\title{
Clinical Role of the Detection of Human Telomerase RNA Component Gene Amplification by Fluorescence in situ Hybridization on Liquid-Based Cervical Samples: Comparison with Human Papillomavirus-DNA Testing and Histopathology
}

\author{
Roberta Zappacosta ${ }^{a, b}$ Manuel Maria lanieric Danilo Buca ${ }^{c}$ Elena Repetti ${ }^{d}$ \\ Alessandra Ricciardulli ${ }^{c}$ Marco Liberati ${ }^{c}$ \\ a Surgical Pathology Unit, 'SS Annunziata Hospital' of Chieti, and 'b School of Clinical Biochemistry and \\ 'Obstetric and Gynaecology Unit, 'G. d'Annunzio' University of Chieti-Pescara, Chieti Scalo, and \\ ${ }^{\mathrm{d}}$ Italian Diagnostic Center, Milan, Italy
}

\section{Key Words}

Cervical cancer prevention - 3 q26 chromosome .

Human telomerase RNA component gene amplification .

Human papillomavirus

\begin{abstract}
Objective: This study was designed to evaluate whether the adjunct of human telomerase RNA component (hTERC) fluorescence in situ hybridization (FISH) analysis to cytological diagnosis and human papillomavirus (HPV)-DNA testing may serve as a predictive marker for distinguishing cervical lesions destined to regress from those at high risk of progression towards invasive cancer. Study Design: hTERC FISH analysis was performed on 54 residual liquid-based cytology specimens obtained from women referred to colposcopy for the detection of atypical squamous cells of undetermined significance or worse (ASCUS+) lesions. Histological diagnosis was considered the gold standard and cervical intraepithelial neoplasia of grade 2 or worse $(\mathrm{CIN} 2+)$ as the worst outcome. Results: Oncogenic HPV-DNA was found in $96.3 \%$ of the specimens. Among these, $38.5 \%$ revealed a $\mathrm{CIN} 2+$ diagnosis. hTERC gene amplification was detected in $37 \%$ of
\end{abstract}

the cases; among these, 70\% showed up as CIN2+. hTERC FISH analysis significantly improves the specificity and positive predictive value of HPV-DNA testing, thus differentiating patients with a CIN2+ diagnosis from those with a CIN2diagnosis. Conclusions: Despite the limitation of a small study sample, our findings provide promising data, indicating the possible role of hTERC analysis in the assessment of the risk of developing cervical cancer. This approach would implement the specificity of DNA testing, avoiding overtreatment at the same time. Prospective follow-up studies are needed with the aim of introducing hTERC FISH into decision-making algorithms.

(c) 2015 S. Karger AG, Basel

Cervical squamous-cell carcinoma (CC) is the secondmost common cancer in women worldwide, with at least 400,000 new cases detected every year [1].

Despite being the first neoplasm for which there is an easy preventive and therapeutic approach, $\mathrm{CC}$ remains an important cause of morbidity and mortality. This is main-

R.Z. and M.M.I. contributed equally to this paper.
Correspondence to: Dr. Roberta Zappacosta

Surgical Pathology Unit, 'SS Annunziata Hospital' of Chiet Via dei Vestini

IT-66100 Chieti Scalo (Italy)

E-Mail zappacosta2@hotmail.com 
ly due to the low sensitivity and poor reproducibility of cytological diagnosis.

The accuracy of the Papanicolaou (Pap) test has been recently improved by the use of liquid-based cytology (LBC), but its diagnostic performance remains lower, especially for certain diagnostic categories such as atypical squamous cells of undetermined significance (ASCUS) and low-grade squamous intraepithelial lesions (LSIL) [2]. As a consequence, the management of women with such lesions still remains controversial [2].

Epidemiological and molecular studies demonstrated that oncogenic human papillomavirus (HPV) infection represents the most significant risk factor for cervical carcinogenesis. CC in the absence of oncogenic HPV is rare [3]. On the other hand, infection with oncogenic HPV types is a necessary but not sufficient condition.

$\mathrm{CC}$ emerges via a series of precursor lesions with an increased degree of severity, classified as cervical intraepithelial neoplasia (CIN) grades 1-3. These lesions retain the capacity to spontaneously regress. The Centers for Disease Control (CDC) calculated that at least $80 \%$ of women will acquire HPV infection during their lives, but only $10 \%$ of these will develop a persistent infection and be at a high risk of progression towards invasive cancer [4].

The introduction of HPV-DNA testing into the diagnostic algorithm of patients with cervical lesions has certainly improved the sensitivity of the Pap test, but the main problem remains, i.e. the high prevalence of HPV infection in comparison with the low number of women developing invasive cancer [5].

The current concept of CC prevention aims to identify patients who would benefit from medical interventions, without concerning individuals who would not benefit. Currently, it is possible to differentiate 3 levels of risk of developing cervical cancer: (1) infection with oncogenic HPV types, (2) the emergence of cell clones with a deregulated expression of viral oncogenes and (3) the progression of a few mutated clones towards a large cell population, and the subsequent increased risk of chromosomal instability [6]. Thus, the ideal predictive biomarker would have the capacity to early identify cell populations which have acquired chromosomal instability and are able to evade the antioncogene host mechanisms [5].

Integration of the HPV genome into the host genome is certainly a key factor leading to CIN progression. There is consensus that integration is common in high-grade $\mathrm{CIN}$ and cancer, but infrequent or lacking in regressing lesions. HPV integration, escaping immune system surveillance, would induce the overexpression of E6 and E7 oncogenes with a consequent accumulation of genetic aberrations [7]. Detection of viral messenger (m)RNA transcripts of E6 and E7 oncoproteins has been found to be a promising approach for the management of women with cervical lesions [7]. Currently, the E6/E7 mRNA test represents the most hopeful test for the identification of cervical lesions with a high risk of progression. However, RNA extraction requires trained human resources and is too labor-intensive for routine application [7].

The deregulated expression of viral E6/E7 oncogenes induces chromosomal instability, resulting in the loss and gain of chromosomal material within the affected cells [8]. One of the most prevalent genomic alterations in HPV-transformed cells involves the long arm of chromosome 3 (3q), which contains the human telomerase RNA component (hTERC) [9]. Telomerases, located at the ends of chromosomes, influence cell replication. They shorten during each cell division and seem to be like a clock for cells [10].

Telomerase consists of two essential components: one is the functional RNA component (in humans called hTERC), which serves as a template for telomeric DNA synthesis; the other is a catalytic protein (hTERT) with reverse transcriptase activity. This complex synthesizes telomeres and stabilizes their length. Mutation in any of these components may result in neoplasia. Telomerase activity is reported to be low in normal human somatic cells [11]. Proliferating cells become senescent when telomeres are shortened or when there is no telomerase activity [11]. Cancer cells overcoming this senescence pathway are immortalized [12]. Immortalization is usually accompanied by abnormal activation of telomerases, which seem to be essential for the progression of neoplastic clones. In view of this, telomerase activity can be used to distinguish between malignant and normal cells [10-12].

The hTERC gene seems to be crucial for the reactivation of telomerases as well as neoplastic transformation by blocking cell apoptosis [12]. For these reasons, hTERC gene amplification has been recently regarded as a marker for predicting prognosis for women with cervical lesions.

This study was designed to determine the correlation between hTERC gene amplification and oncogenic HPV infection, and to assess the potential usefulness of this genomic marker for identifying cervical lesions which are more likely to persist and induce invasive cancer. To do this, this fluorescence in situ hybridization (FISH) technique was used to retrospectively detect the hTERC on exfoliated cells collected from the cervix on behalf of the regional Cervical Cancer Screening Program.
Zappacosta/Ianieri/Buca/Repetti/ Ricciardulli/Liberati 


\section{Material and Methods}

\section{Selection of Patients}

This study was performed according to the principles outlined in the Declaration of Helsinki of 1975, and it was approved by the local editorial board.

Case series were extracted from the electronic database of the outpatient department of 'ASL 2 Abruzzo', by analyzing the clinical history of patients who underwent colposcopy and colposcopydirected biopsy from January 2012 to July 2013. We selected patients referred to colposcopy for ASCUS+ cytological diagnosis. An additional inclusion criterion was the availability (for each patient) of a residual liquid-based cervical specimen collected for cytological diagnosis.

Exclusion criteria were: treatment for cervical lesion in the past 5 years, a history of any type of cancer, surgical or ablative treatment (except for biopsy) during baseline colposcopy, hysterectomy, HIV positivity or other causes of immunodeficiency and pregnancy.

Written informed consent was obtained from the patients and an identification code was assigned to each case, upholding standards of confidentiality.

The LBC result was reported according to the 2001 revision of the Bethesda System [13], as ASCUS, LSIL, high-grade squamous intraepithelial lesion (HSIL) and CC.

Histological diagnosis was classified as normal or benign cellular changes (NILM), CIN1-3 and invasive squamous-cell carcinoma, according to the $2005 \mathrm{WHO}$ guidelines [14]. Two independent surgical pathologists, separately and blinded to all other study results, revised tissue slides and established the final diagnosis, basing it on the highest CIN grade detected on each sample.

We assumed that both the colposcopy and biopsy were 100\% sensitive. In view of this, histological diagnosis from colposcopydirected biopsies was accepted as a verification of disease status and regarded as the gold standard. NILM and CIN1 are referred to here as less severe than CIN2 (CIN2-), and CIN2, CIN3 and CC as CIN2+. CIN2+ was regarded as the worst outcome.

Only patients for whom there was consensus about the histological diagnosis were finally included in the study.

\section{$L B C$ and $H P V-D N A$ Test}

Cervicovaginal samples were collected from the ecto- and endocervix with an Ayre spatula and a Cytobrush. Cytological specimens were then directly transferred in ThinPrep vials (ThinPrep ${ }^{\circledR}$, Hologic, Marlborough, Mass., USA) containing $20 \mathrm{ml}$ of PreservCyt solution.

To prepare cytological slides from liquid-based cervical samples, a ThinPrep 5000 processor (Hologic Corp., Boxborough, Mass., USA) was used. Slides were then stained with a Pap procedure.

An aliquot $(4 \mathrm{ml})$ of each $\mathrm{LBC}$ sample, stored at room temperature, was removed for the detection of oncogenic HPV-DNA by the Digene Hybrid Capture 2 method (HC2, Qiagen, Gaithersburg, Md., USA). A total of 13 oncogenic HPV types were detected $(16,18,31,33,35,39,45,51,52,56,58,59$ and 68$)$. The test was performed according to the manufacturer's instructions. HC2 reactions, as chemiluminescent signals, were then read by the offline luminometer system (Digene microplate luminometer, DML 2000), which provided relative quantification of each sample in comparison to the mean of a series of positive controls containing
$1 \mathrm{pg} / \mathrm{ml}$ of HPV-DNA (corresponding to approx. 100,000 HPV-16 genomes/ml or 5,000 HPV copies/reaction). A cut-off value of 1 relative light unit, corresponding to $>1 \mathrm{pg} / \mathrm{ml}$ of HPV-DNA, was used to classify the specimen as positive.

\section{Amplification of hTERC Gene by FISH}

In the laboratories of the Italian Diagnostic Center of Milan, residual liquid-based cervical samples were also used to perform hTERC FISH analysis (Kreatech Diagnostics, Amsterdam, The Netherlands). FISH probes containing the hTERC gene at locus $3 q 26.2$ in spectrum yellow and a control probe at chromosome 7 in spectrum blue were applied.

Cervical cells, sorted and adherent to a glass slide, were subjected to a temperature of $70^{\circ} \mathrm{C}$ for $5 \mathrm{~min}$ to denature the DNA molecules. A specific probe for the $3 \mathrm{q} 26$ region, conjugate with yellow dye gold, was applied on each slide. Hybridization with a hTERC (3q26) probe was carried out at $37^{\circ} \mathrm{C}$ for $14 \mathrm{~h}$. After hybridization, slides were washed with the first post-hybridization wash buffer, at $73^{\circ} \mathrm{C}$ for $2 \mathrm{~min}$, and with the second post-hybridization wash buffer at room temperature for $1 \mathrm{~min}$.

In order to contrast the yellow gold, nuclear chromatin was stained with DAPI blue dye. Upon completion of the bond between the probe and its complementary region, the chromosome was labeled and highlighted by the binding fluorescence.

Evaluation of the hTERC FISH results was performed blinded to the cytological and histological diagnosis and clinical data. Slides were viewed at $\times 1,000$ magnification on a fluorescence microscope equipped with Spectrum Gold and DAPI filters, by 2 independent observers. In the case of discrepancies, the results were discussed with a third observer until a consensus was reached.

The dark background of the nucleus of the cells appeared as blue due to DAPI staining of the chromatin and the hTERC gene locus appeared as yellow gold. The number of signals for hTERC and chromosome 7 were evaluated per nucleus. In normal cells, each nucleus contains only 2 copies of the telomerase gene (corresponding to 2 yellow spots) and 2 of chromosome 7 (corresponding to 2 blue spots). Thus, the expected ratio in the normal cells was 2:2. Cells with the telomerase gene amplification would contain multiple copies of the 3 q26 gene.

In this study and for each cytological specimen, 2,000 cells were scored for an abnormal signal of both 3q26 and centromeric chromosome 7 . To avoid counting split signals as 2 signals, the distance between any 2 signals had to be at least the diameter of 1 signal, in order for them to be counted as individual signals. A case was considered positive for $3 \mathrm{q} 26$ amplification by having $\geq 5$ locus-specific signals in at least $10 \%$ of the abnormal cells according to the criteria of Obermann et al. [15]. Image acquisition and analysis were performed using Ikoniscope digital microscopy system automation (Konisys Inc., New Haven, Conn., USA) equipped with optical filters.

\section{Statistical Analysis}

We calculated the prevalence of HPV-DNA and hTERC FISH positivity using standard methods.

In order to correlate HPV-DNA and FISH results with histological diagnosis, $2 \times 2$ tables were used. Sensitivity and specificity of each test individually as well as in comparison to one another were assessed by receiver operating characteristic (ROC) analysis, with the histological diagnosis as the gold standard. Areas under the ROC curves with $95 \%$ confidence intervals (CIs) were estimat- 
Table 1. Summary of results from cytological and histological diagnosis, HPV-DNA testing and hTERC FISH analysis

\begin{tabular}{|c|c|c|c|c|c|c|c|c|}
\hline $\begin{array}{l}\text { Cytological } \\
\text { diagnosis }\end{array}$ & NILM & CIN1 & CIN2 & CIN3 & positive & negative & positive & negative \\
\hline LSIL & $3(37.5)$ & $17(65.4)$ & $2(22.2)$ & $3(27.3)$ & $7(35)$ & $18(53)$ & $25(48.1)$ & 0 \\
\hline HSIL & 0 & 0 & 0 & $6(54.5)$ & $5(25)$ & $1(2.9)$ & $6(11.5)$ & 0 \\
\hline
\end{tabular}

Values are expressed as $\mathrm{n}(\%)$

ed to assess differences in performance, and the McNemar test was used for statistical significance. Positive and negative predictive values (PPV and NPV) were also calculated, and the results were given with $95 \%$ CIs.

The agreement of the HPV-DNA test and FISH analysis with the histological diagnosis was calculated by kappa statistics. According to the criteria of Landis and Koch [16], the $\kappa$ values were divided into 6 scales of strength of agreement: poor $(<0.00)$, slight $(0.00-0.20)$, fair $(0.21-0.40)$, moderate $(0.41-0.60)$, substantial $(0.61-0.80)$ or almost perfect $(0.81-1.00)$ [16].

Odds ratios (ORs) were used to correlate both the HPV-DNA and FISH results with the histological outcome. All statistical analyses were performed with SPSS software v15.0 (SPSS for Windows, SPSS Inc., Chicago, Ill., USA). In all analyses, $\mathrm{p}<0.05$ was regarded as significant.

\section{Results}

The study included 54 patients (mean age $35.7 \pm 10.8$, median 34 and range $22-65$ years). Table 1 shows the distribution of patients according to the cytological and histological diagnoses, the HPV-DNA test and the hTERC analysis.

\section{hTERC and Cytological Categories}

We detected 23 ASCUS (42.6\%), 25 LSIL (46.3\%) and 6 HSIL (11.1\%) cases. The PPV of the different cytological categories was $39.1 \%$ for ASCUS, $20 \%$ for LSIL and $100 \%$ for HSIL, respectively. The corresponding NPV was 60.9, 80 and $100 \%$, respectively $(\mathrm{p}<0.05)$. hTERC FISH showed a clear signal in $100 \%$ of the specimens (fig. 1). hTERC gene amplification was found in 37\% (20/54) of the cases.

In the ASCUS group, a positive result was detected on FISH in $34.8 \%(8 / 23)$ of the cases. Among these, $75 \%$ $(6 / 8)$ were CIN2+ and 25\% (2/8) were CIN2- $(\mathrm{p}<0.0001)$. In the LSIL category, hTERC gene amplification was detected in $28 \%(7 / 25)$ of the cases. Among these, $42.9 \%$
$(3 / 7)$ were CIN2+ and $57.1 \%(4 / 7)$ were CIN2- $(\mathrm{p}=0.2)$. Of the HSIL cases, $83.3 \%$ showed hTERC gene amplification and all of these were CIN2+ (CIN3).

Overall, the mean number of cells with hTERC gene amplification was 45.8 (range 2-500). According to the cytological categories, a mean number of 47.5 cells was detected in the ASCUS category, 67.6 in LSIL and 39.6 in HSIL, but the difference did not reach statistical significance ( $\chi^{2}$ test; $\mathrm{p}=0.22$ ).

Table 2 correlates the positive hTERC result with the cytological and histological diagnoses. Within the ASCUS category, hTERC analysis differentiated patients histologically diagnosed as CIN2+ (66.7\%) from those diagnosed as CIN2- (14.3\%) ( $\mathrm{p}<0.0001)$. Likewise, hTERC gene amplification differentiated LSIL resulting as CIN2+ (60\%) from those revealing CIN2- (20\%) (p < $0.0001)$.

The sensitivity, specificity, PPV and NPV of the hTERC analysis, with CIN2+ as the gold standard, were 66.7, 85.7, 75 and 80\% for ASCUS, and 60, 80, 42.9 and $88.9 \%$ for LSIL, respectively. The sensitivity and PPV for HSIL were 75 and $100 \%$, respectively. No CIN2- were detected in the HSIL group. hTERC FISH analysis significantly improved the PPV of the ASCUS diagnosis $(\mathrm{p}<0.01)$, but for the LSIL and HSIL categories, the difference did not reach statistical significance.

\section{Correlation between hTERC and Histological Diagnosis}

There were 8 patients with NILM (14.8\%), 26 CIN1 cases (48.1\%), 9 CIN2 cases (16.7\%) and 11 CIN3 cases (20.4). No SCC was detected. The overall CIN2+ prevalence was $37 \%$ (20/54 cases); CIN2+ was detected in $39.1 \%$ (9/23) of the ASCUS cases, 20\% (5/25) of the LSIL cases and $100 \%(6 / 6)$ of the HSIL cases. Among the positive hTERC cases, $70 \%(14 / 20)$ were CIN2+ and 30\% (6/20) 
Fig. 1. Thin-layer slides from liquid-based specimens. Pap. $\times 20$ and corresponding hTERC dual-color FISH, $\times 20$. hTERC gene $=$ yellow fluorescence and chromosome $7=$ blue fluorescence. a ASCUS. b Normal 2:2 hTERC signal pattern. c LSIL. d Abnormal 6:2 hTERC signal pattern. e HSIL. f Abnormal 5:4 hTERC signal pattern.
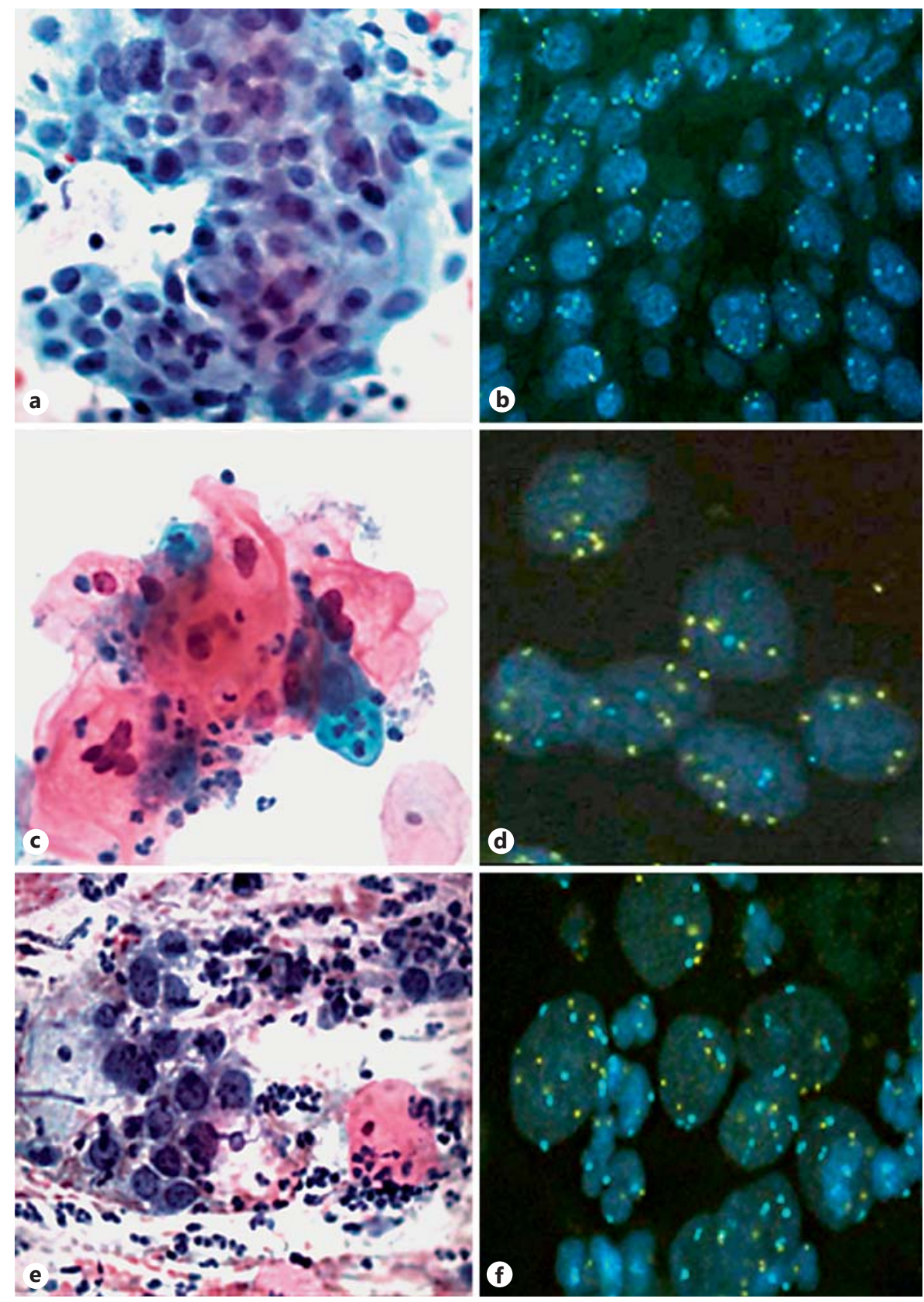

were CIN2-. Among the negative FISH cases, $82.4 \%$ $(28 / 34)$ showed CIN2- and $17.6 \%$ CIN2+ $(\mathrm{p}<0.05)$.

Upon considering the histological categories as distinct, no NILM showed FISH amplification, while $23.1 \%(6 / 26)$ with CIN1, 66.7\% (6/9) with CIN2 and 72.7\% (8/11) with CIN3 tested as hTERC positive. The proportion of hTERC gene amplification significantly increased from CIN1 to CIN3 (Cochran-Armitage test for trend; $\mathrm{p}<0.0001$ ).
The percent agreement between the FISH results and the histological diagnosis was $77.8 \%$ (Cohen's $\kappa$ value: 0.52 ). The sensitivity, specificity, PPV and NPV of hTERC FISH were $70,82.4,70$ and $82.3 \%$, respectively. A positive FISH result was associated with a CIN2+ diagnosis (OR $2.18,95 \%$ CI 1.11-4.28; $\mathrm{p}=0.02$ ). These data indicate the significant association between hTERC gene amplification and a CIN2+ diagnosis. 


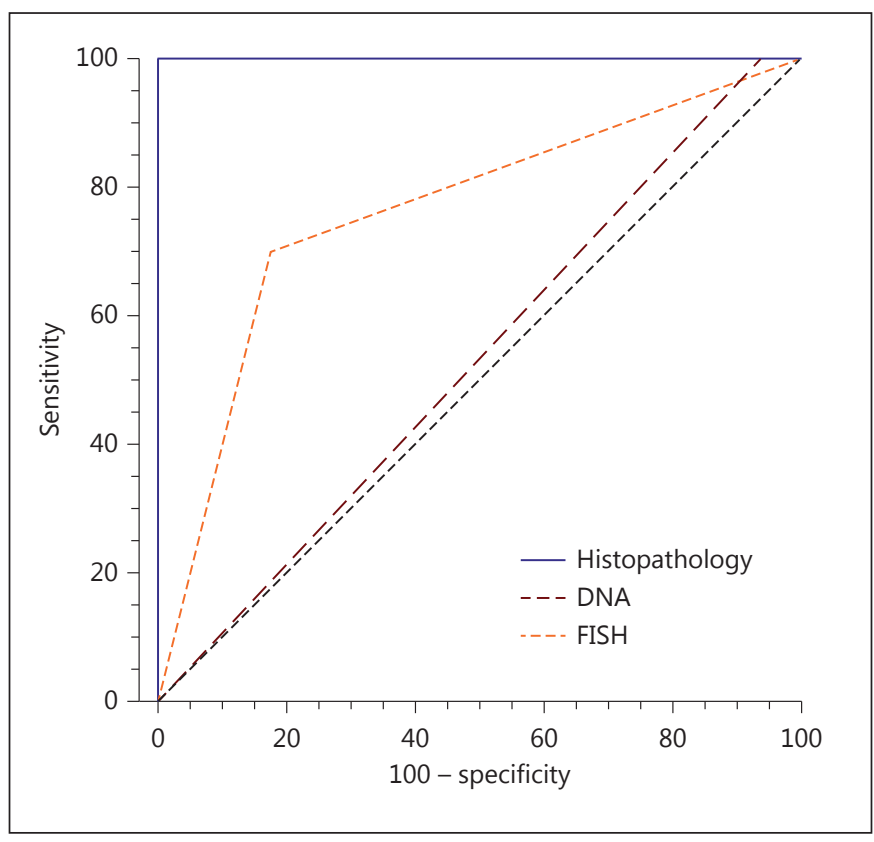

Fig. 2. ROC curves comparing the sensitivity and specificity of HPV-DNA and hTERC FISH with the corresponding parameters of histological diagnosis $(\mathrm{p}=0.04)$. Bisector line indicates a reference threshold with an AUC of 0.5.

\section{hTERC and HPV-DNA Test}

HPV-DNA testing showed a positive result in 52/54 $(96.3 \%)$ of the cases, particularly in $21 / 23(91.3 \%)$ of ASCUS, 25/25 (100\%) of LSIL and 6/6 (100\%) of HSIL cases. The proportion of DNA-positive results increased with the severity of cytological lesion (Cochran-Armitage test for trend; $\mathrm{p}=0.13$ ).

All (20/20) of the CIN2+ and 94.1\% (32/34) of the CIN2- patients revealed a HPV-DNA-positive result. Moreover, $87.5 \%$ of NILM resulted as DNA positive. A positive DNA test result conferred an OR for CIN2+ of 0.63 (95\% CI 0.04-10.57; $\mathrm{p}=0.7$ ). Overall percent agreement between DNA testing and histological diagnosis was $40.7 \%$ (Cohen's $\kappa$ value: 0.04 ).

The overall sensitivity, specificity, PPV and NPV for DNA testing were 100\% (95\% CI 83.8-100), 5.9\% (95\% CI 1.63-19.1), 38.5\% (95\% CI 23-51) and 100\% (95\% CI 83.8-100), respectively.

The corresponding values for the hTERC FISH analysis were $70 \%$ (95\% CI 47.8-85.5), 82.4\% (95\% CI 6691.9), 70\% (95\% CI 49.9-90.1) and 82.3\% (95\% CI 69.595.2), respectively. The specificity and PPV of the DNA test were significantly improved by the hTERC FISH analysis (McNemar test; $\mathrm{p}<0.0001)$. ROC analysis for ac-
Table 2. hTERC amplification according to the cytological and histological diagnoses

\begin{tabular}{llll}
\hline \multirow{2}{*}{ Histology } & \multicolumn{3}{l}{ Cytology, $\mathrm{n}(\%)$} \\
\cline { 2 - 4 } & ASCUS $(\mathrm{n}=23)$ & LSIL $(\mathrm{n}=25)$ & HSIL $(\mathrm{n}=6)$ \\
\hline NILM & $0 / 5(0)$ & $0 / 3(0)$ & $0 / 0(0)$ \\
CIN1 & $2 / 9(22.2)$ & $4 / 17(23.5)$ & $0 / 0(0)$ \\
CIN2 & $5 / 7(71.4)$ & $1 / 2(50)$ & $0 / 0(0)$ \\
CIN3 & $1 / 2(50)$ & $2 / 3(66.7)$ & $5 / 6(83.3)$ \\
\hline
\end{tabular}

Table 3. Correlation between HPV-DNA test and hTERC FISH analysis

\begin{tabular}{lccc}
\hline HPV-DNA test & \multicolumn{4}{l}{ hTERC FISH analysis } \\
\cline { 2 - 4 } & positive & negative & all \\
\hline Positive & $19(95)$ & $33(97.1)$ & $52(96.3)$ \\
Negative & $1(5)$ & $1(2.9)$ & $2(3.7)$ \\
\hline All & $20(37)$ & $34(63)$ & 54 \\
\hline
\end{tabular}

Values are expressed as $\mathrm{n}(\%)$.

curacy showed an area under the curve (AUC) reaching 0.53 (95\% CI 0.39-0.67) for the DNA test and 0.76 (95\% CI 0.63-0.87) for FISH, respectively. Histological diagnosis, as the gold standard, showed an AUC of 1 . Differences were statistically significant ( $p<0.05$; fig. 2 ).

To investigate the relationship between HPV infection and hTERC gene amplification, we analyzed both tests as combined. Not all genetically abnormal cases were diagnosed as HPV positive, and not all HPV-positive cases showed hTERC gene amplification. There was oncogenic HPV infection in 95\% (19/20) of positive hTERC cases (McNemar test; $\mathrm{p}<0.05$; table 3 ). HPV-DNA/hTERC positivity increased along with the degree of cervical dysplasia $(\mathrm{p}<0.001)$.

\section{Discussion}

The Pap test is unanimously accepted as the most costeffective screening tool for detecting precancerous cervical disease. Thanks to the wide use of cytological testing, cervical cancer incidence is kept under control in the socalled industrialized countries, and the prevalence of relevant lesions in the population is currently very low. 
In spite of this, cytological screening has some limitations, including the low sensitivity and the poor interobserver reproducibility of morphological interpretation. An audit of UK National Health Service Cancer Screening Programmes on cervical cancer found that CC occurred in $29 \%$ of women under 65 years of age, whose screening was up to date and in line with national guidelines [17]. ASCUS diagnosis, which is considered the borderline between clearly normal and clearly abnormal, is pathognomonic in this sense, underlying $38.8 \%$ of histologically confirmed high-grade lesions [18-21]. On the other hand, only $10 \%$ of cervical lesions are at a high risk for progression towards invasive cancer [22]. This fact is particularly true for low-grade cervical abnormalities, the management of which is not yet clearly defined, ranging from repetition of cytology at shorter intervals to immediate referral to colposcopy [18]. In summary, an LSIL diagnosis would often cause overtreatment and extensive costs [19], while patients with an ASCUS diagnosis would have a small but significant risk of progression toward high-grade lesions [20].

To improve the diagnostic accuracy of cytology, many tests have been evaluated, such as HPV-DNA test and p16 ${ }^{\text {INK4a }}$. The American Society for Colposcopy and Cervical Pathology recommends to triage patients with ASCUS and LSIL with HPV-DNA assay [23, 24], but the transitory nature of HPV infection would confer a low specificity to the HPV-DNA test for detecting high-grade intraepithelial neoplasia or invasive cancer. For this reason, the scientific community is looking for a supplemental test that would bypass this issue and provide information on the regression or progression of cervical lesions.

Several factors, such as a loss of cellular inhibiting factors and the integration of the HPV genome into the host genome, can lead to the expression of E7 in the basal cell layers. The result is cellular transformation and a massive accumulation of $\mathrm{p} 16^{\mathrm{INK} 4 \mathrm{a}}$ in the cells [25]. The usefulness of the immunocytochemical detection of p16 in cervical samples has been analyzed in many trials, particularly as the triage test for ASCUS and LSIL. While the sensitivity of p16 in detecting underlying CIN2+ was similar to that of HPV testing, its specificity was confirmed to be higher [26]. On the other hand, a recent meta-analysis showed a substantial variability in the literature regarding the cutoff for positive p16 results [27]. This variability is probably due to the wide range of color-based qualitative (i.e. nuclear or cytoplamatic positivity and staining intensity) and quantitative parameters (i.e. the number of immunoreactive cells) which (singly or in combination) are usually used. The purely color-based approach to identify

Detection of FISH hTERC Amplification on Liquid-Based Cervical Specimens abnormal cells in cervical smears using p16, is hampered by the fact that some normal endocervical, endometrial, intercalated, squamous metaplastic or atrophic cells may also display p16 immunoreactivity. The use of a 4-tiered classification score has been proposed [28]. However, due to the high variability when relying on p16 interpretation and despite the relatively low cost of this immunohistochemical assay (EUR 57 vs. 81.60 for DNA testing and EUR 164.55 for hTERC FISH according to the Italian NHS tariff), there is still disagreement about the use of p16 as a predictive marker in clinical practice.

Several studies postulated the essential role of $3 q 26$ during the progression of cervical lesions to invasive cancer [24], but the application of molecular techniques able to identify hTERC gene amplification in a clinical setting is still evolving [29-33]. Our goal was to evaluate $3 q 26$ hTERC FISH analysis on liquid-based cervical samples, in order to establish its accuracy as a predictive test and its feasibility for routine application.

Our study differs from previous reports $[34,35]$. To our knowledge, this is the first time that hTERC FISH analysis has been performed as a reflex test on residual LBC specimens which have also been used for cytological diagnosis and HPV-DNA testing. Moreover, the inclusion of patients with varying degrees of cytological abnormalities made our setting different from the study by Ying et al. [36], who conducted their analyses in an HPVDNA-positive population exhibiting ASCUS and LSIL.

The percentage of successfully hybridized samples was $100 \%$ in our study, considerably higher than in other studies [30-33]. In keeping with previous studies, we found a significant correlation (95\%) between the HPVDNA test and hTERC FISH. This finding would confirm the possible role of HPV in the amplification of the hTERC gene [34]. Like Branca et al. [35], we found a linear relationship between higher grades of CIN and the percentage of cervical samples in which hTERC amplification was found. Our data showed that extra copies of the hTERC gene were present in about $23 \%$ of CIN1, $66.7 \%$ of CIN 2 and $72.7 \%$ of CIN 3 cases, but were lacking in nonpathological tissue. Moreover, the hTERC analysis significantly differentiated the CIN2- from the CIN2+ cases. Branca et al. [35], on the other hand, evaluated the expression of hTERC on histological specimens by immunohistochemical assay, grading the nuclear staining into 4 categories. Immunohistochemistry demonstrates high feasibility in the clinical setting, but its use for hTERC assessment is sometimes difficult due to special conditions under which nondysplastic cells also stain positive. In such cases, the evaluation of hTERC-positive cells re- 
quires subjective interpretation of both cellular and nuclear morphology, and would possibly lead to poor reproducibility of results.

Considering the cytological categories, we demonstrated that patients with an HSIL diagnosis had a significantly higher rate of hTERC gene amplification than patients with ASCUS and LSIL.

Among the ASCUS and LSIL cases, hTERC analysis enables significant differentiation between patients with CIN2+ and CIN2-, thereby improving the PPV of such cytological categories. These findings, comparable with previous studies, strongly support the potential clinical usefulness of this genetic test for the correct management of women with borderline/low-grade cervical abnormalities [36-40].

ROC curves, integrating sensitivity and specificity for a diagnostic test, would supply a measure of its accuracy. The AUC, in particular, has proved to be a powerful tool to measure the ability of a test to correctly classify patients with or without disease. In our study, the ROC curves demonstrated the higher accuracy of hTERC FISH for the detection of CIN2+ in comparison to that of DNA test.

We found that $87.5 \%$ of NILM cases tested HPV-DNA positive, even though the study population consisted of women with a mean age of 35 years. There are several possible explanations for this. First, there is the specific limitation of the HC2 technique; it does not include internal control for quality or performance. Thus, the presence of a very high load of nononcogenic HPV-DNA in the sample, as we found, would give rise to false-positive results for oncogenic genotypes by cross-hybridization [41]. This is certainly a considerable problem in populations with a high predominance of infections induced by these viral genotypes. Pathognomonic in this sense is the paper by Adams et al. [42], in which $12.2 \%$ of the cases with an ASC diagnosis revealed an oncogenic HPV infection by $\mathrm{HC} 2$ and a negative cervical biopsy result.

Another reasonable explanation for a negative biopsy result in women with oncogenic HPV infection would be the sampling error that occurred during colposcopic examination [42]. This hypothesis would underline the importance of the use of predictive markers during followup of HPV-positive patients. Finally, the substantial number of cases in our study that had a positive HPVDNA test associated with a negative histological diagnosis might be also related to the natural history of the HPV infection itself. HPV infection can be transient, especially but not exclusively, in women $\leq 30$ years of age. The probability of spontaneous resolution is high within the sec-

ond year [43]. About $43 \%$ of the patients in our study with a negative biopsy but an HPV-positive result were $<30$ years old, but most of them underwent biopsy within 1 year of the index HPV-DNA test. For all the reasons listed above, hTERC FISH analysis appears to be a promising discriminating tool, able to distinguish cervical lesions on the basis of their progression potential.

Finally, there are some considerations about the feasibility of the hTERC FISH test in clinical practice. hTERC FISH technology is similar to that used for UroVision, which is widely utilized to analyze urine specimens. We strongly believe that advances in cervical LBC and automation during FISH procedure would allow for the routine use of hTERC analysis in clinical settings. In particular, we recommend both HPV and FISH testing on the same vial. In view of this, the ability to perform multiparametric analysis on the same cervical specimens would certainly lead to more effective CC prevention strategies.

In our opinion, the combination of the HPV-DNA test and hTERC FISH analysis can serve as an important application for the management of women with borderline/ mild cervical abnormalities [36]. We are aware that our study had some limitations, such as the small number of cases. To obviate this drawback, further prospective investigations with long-term follow-up will be necessary, in view of the possible introduction of hTERC FISH analysis into decision-making algorithms [44-50].

\section{Disclosure Statement}

The authors declare no conflicts of interest.

References

Acta Cytologica 2015;59:345-354 DOI: $10.1159 / 000438719$
Zappacosta/Ianieri/Buca/Repetti/ Ricciardulli/Liberati
1 Arbyn M, Castellsague X, de Sanjose S, Bruni L, Saraiya M, Bray F, Ferlay J: Worldwide burden of cervical cancer in 2008. Ann Oncol 2011;22:2675-2686.

-2 American Congress of Obstetricians and Gynecologists (ACOG): Practice Bulletin No. 131: screening for cervical cancer. Obstet Gynecol 2012;120:1222-1238.

- 3 Bosch FX, Lorincz A, Munoz N, Meijer CJLM, Shah KV: The causal relation between human papillomavirus and cervical cancer. J Clin Pathol 2002;55:244-265.

4 Arbyn M, Buntinx F, Van Ranst M, Paraskevaidis E, Martin-Hirsch P, Dillner J: Virologic versus cytologic triage of women with equivocal Pap smears: a meta-analysis of the accuracy to detect high-grade intraepithelial neoplasia. J Natl Cancer Inst 2004;96:280-293. 
5 Petry KU, Mention S, Menton M, van LoenenFrosch F, de Carvalho Gomes H, Holz B, et al: Inclusion of HPV testing in routine cervical cancer screening for women above 29 years in Germany: results for 8,468 patients. Br J Cancer 2003;88:1570-1577.

-6 Szarewski A, Ambroisine L, Cadman L, Austin J, Ho L, Terry G, et al: Comparison of predictors for high-grade cervical intraepithelial neoplasia in women with abnormal smears. Cancer Epidemiol Biomarkers Prev 2008; 17 : 3033-3042.

7 Kraus I, Molden T, Holm R, Lie AK, Karlsen F, Kristensen GB, et al: Presence of E6 and E7 mRNA from human papillomavirus types 16 , $18,31,33$, and 45 in the majority of cervical carcinomas. J Clin Microbiol 2006;44:13101317.

-8 Matthews CP, Shera KA, McDougall JK: Genomic changes and HPV type in cervical carcinoma. Proc Soc Exp Biol Med 2000;223: 316-321.

9 Cayuela L, Flores JM, Blasco MA: The telomerase RNA component TERC is required for the tumour-promoting effects of TERC overexpression. EMBO Rep 2005,6:268-274.

10 Farwell DG, Shera KA, Koop JI, Bonnet GA, Matthews CP, Reuther GW, Coltreta MD, McDougall JK, Klingelhutz AJ: Genetic and epigenetic change in human epithelial cells immortalized by telomerase. Am J Pathol 2000;156:1537-1544.

11 Anderson S, Shera K, Ihle J, Billman L, Goff B, Greer B, Tamimi H, McDougall J, Klingelhutz A: Telomerase activation in cervical cancer. Am J Pathol 1997;151:25-31.

12 Hills M, Lansdorp PM: Short telomeres resulting from heritable mutations in telomerase reverse transcriptase gene predispose for a variety malignancies. Ann NY Acad Sci 2009;1176:178-190.

13 Solomon D, Davey D, Kurman R, Moriarty A, O'Connor D, Prey M: The 2001 Bethesda System: terminology for reporting results of cervical cytology. JAMA 2002;287:2114-2119.

14 World Health Organization: IARC Handbooks of Cancer Prevention. Vol 10. Cervix Cancer Screening. Lyon, IARC Press, 2005, pp 1-302.

15 Obermann EC, Prince SS, Barascud A, Grilli B, Herzog M, Kaup M, Cathomas G, Frey Tirri B, et al: Prediction of outcome in patients with low-grade squamous intraepithelial lesions by fluorescence in situ hybridation analysis of human papillomavirus, TERC, and Myc. Cancer Cytopathol 2013;8:423-431.

16 Landis JR, Koch GG: The measurement of observer agreement for categorical data. Biometrics 1977;1:159-174.

17 NHSCSP Audit of Invasive Cervical Cancer: national report 2007-2010. www.cancerscreening.nhs.uk/cervical/publications/nhscsp-audit-invasive-cervicalcancer-201107.pdf (last accessed January 9, 2014).
8 Cuschieri KS, Graham C, Moore C, Cubie HA: Human papillomavirus testing for the management of low-grade cervical abnormalities in the UK. Influence of age and testing strategy. J Clin Virol 2007;38:14-18.

19 Arbyn M, Kyrgiou M, Simoens C: Peri-natal mortality and other severe adverse pregnancy outcome associated with treatments of cervical intraepithelial neoplasia: a meta-analysis. BMJ 2008;337:1284-1295.

20 Massad LS, Collins YC, Meyer PM: Biopsy correlates of abnormal cervical cytology classified using the Bethesda System. Gynecol Oncol 2001;82:516-522.

21 Castle PE, Gravitt PE, Wentzensen N, Schiffman M: A descriptive analysis of prevalent vs. incident cervical intraepithelial neoplasia grade 3 following minor cytologic abnormalities. Am J Clin Pathol 2012;138:241-246.

22 Centers for Disease Control and Prevention. www.cdc.gov/std/stats/stiestimates-factsheet-feb-2013.pdf (last accessed December 28, 2014).

23 Atkins KA, Jeronimo J, Stoler MH, et al: Description of patients with squamous cell carcinoma in the Atypical Squamous Cells of Undetermined Significance/Low-Grade Squamous Intraepithelial Lesion Triage Study. Cancer 2006;108:212-221.

24 Wright TC, Massad LS, Dunton CJ, Spitzer M, Wilkinson EJ, Solomon D: 2006 Consensus guidelines for the management of women with abnormal cervical cancer screening tests. Am J Obstet Gynecol 2007;197:346-355.

-25 Lambert AP, Anschau F, Schmitt VM: $\mathrm{P} 16^{\mathrm{INK} 4 \mathrm{a}}$ expression in cervical premalignant and malignant lesions. Exp Mol Pathol 2006,80:192-196.

26 Denton KJ, Bergeron C, Klement P, Trunk MJ, Keller T, Ridder R; European CINtec Cytology Study Group: The sensitivity and specificity of p16 ${ }^{\text {INK4a }}$ cytology vs HPV testing for detecting high-grade cervical disease in the triage of ASC-US and LSIL Pap cytology results. Am J Clin Pathol 2010;134:12-21.

27 Tsoumpou I, Arbyn M, Kyrgiou M, et al: p1 $16^{\text {INK4a }}$ immunostaining in cytological and histological specimens from the uterine cervix: a systematic review and meta-analysis. Cancer Treat Rev 2009;35:210-220.

28 Wentzenses N, Bergeron C, Cas F, Eschenbach D, Vinokurova S, von Knebel DM: Evaluation of a nuclear score for $\mathrm{p} 16^{\mathrm{INK} 4 \mathrm{a}}$-stained cervical squamous cells in liquid-based cytology samples. Cancer 2005;105:461-467.

29 Yang YC, Shyong WY, Chang MS, Chen YJ, Lin $\mathrm{CH}$, Huang ZD, Wang Hsu MT, Chen ML: Frequent gain of copy number on long arm of chromosome 3 in human cervical adenocarcinoma. Cancer Genet Cytogenet 2001;131:48-53.

30 Zhang Y, Wang X, Ma L, Hu L: Clinical significance of hTERC gene amplification detection by FISH in the screening of cervical lesions. J Huazhong Univ Sci Technolog Med Sci 2009;29:368-371.
31 Alemada F, Espinet B, Corzo C, Munoz R, Bellosillo B, Lloveras B, Pijuan L, Gimeno J, Salido M, Solè F, Carreras R, Serrano S: 3q26 (hTERC) gain studied by fluorescence in situ hybridization as a persistence-progression indicator in low-grade squamous intraepithelial lesion cases. Hum Pathol 2009;40:1474-1478.

32 Heselmeyer-Haddad K, Janz V, Castle PE, Chaudhri N, White N, Wilber K, Morrison LE, Auer G, Burroughs FH, Sherman ME, Ried T: Detection of genomic amplification of human telomerase gene (TERC) in cytologic specimens as a genetic test for the diagnosis of cervical dysplasia. Am J Pathol 2003;163: 1405-1416.

33 Heitman ER, Lankachandra KM, Wall J, et al: $3 q 26$ amplification is an effective negative triage test for LSIL: a historical prospective study. PLoS One 2012;7:1-7.

- 34 Hopman AH, Theelen W, Hommelberg PP, Kamps MA, Herrington CS, Morrison LE, Speel EJ, Smedts F, Ramaekers FC: Genomic integration of oncogenic HPV and gain of human telomerase gene TERC at 3q26 are strongly associated events in the progression of uterine cervical dysplasia to invasive cancer. J Pathol 2006;210:412-419.

35 Branca M, Giorgi C, Ciotti M, et al: Upregulation of telomerase (hTERC) is related to the grade of cervical intraepithelial neoplasia, but is not an independent predictor of high-risk human papilloma virus, virus persistence, or disease outcome in cervical cancer. Diagn Cytopathol 2006;34:739-748.

36 Ying L, Wen-Jie Z, Feng Y, Xin-Yu W, WeiGuo L, Ding M, et al: Application of hTERC in ThinPrep samples with mild cytologic abnormality and HR-HPV positive. Gynecol Oncol 2011;120:73-83.

37 Caraway NP, Khanna A, Dawlett M, Guo M, Guo N, Lin E, Katz RL: Gain of the 3q26 region in cervicovaginal liquid-based Pap preparations is associated with squamous intraepithelial lesions and squamous cell carcinoma. Gynecol Oncol 2008;110:37-42.

38 Heselmeyer-Haddad K, Sommerfeld K, White NM, Chaudhri N, Morrison LE, Palanisamy N, Wang ZY, Auer G, Steinberg W, Ried T: Genomic amplification of the human telomerase gene (TERC) in Pap smears predicts the development of cervical cancer. Am J Pathol 2005;166:1229-1238.

39 Reza Jalali G, Herzog TJ, Dziura B, Walat R, Kilpatrick MW: Amplification of the chromosome 3q26 region shows high negative predictive value for non-malignant transformation of LSIL cytologic findings. Am J Obstet Gynecol 2010;202:581-583.

40 Liu Z, Ma H, Wei S, Li G, Sturgis EM: Telomere length and TERT functional polymorphisms are not associated with risk of squamous cell carcinoma of the head and neck. Cancer Epidemiol Biomarkers Prev 2011;20: 2642-2648. 
41 de Cremoux P, Coste J, Sastre-Garau X, et al: Efficiency of the hybrid capture 2 HPV DNA test in cervical cancer screening: a study by the French Society of Clinical Cytology. Am J Clin Pathol 2003;120:492-499.

42 Adams AL, Eltoum I, Roberson J, et al: Negative colposcopic biopsy after positive human papilloma virus (HPV) DNA. Am J Clin Pathol 2006;125:413-418.

43 Molano M, van den Brule A, Plummer M, et al: Determinants of clearance of human papillomavirus infections in Colombian women with normal cytology: a population-based, 5-year follow-up study. Am J Epidemiol 2003; 158:486-494.

-44 Jin G, Yoo SS, Cho S, Jeon HS, Lee WK: Dual roles of a variable number of tandem repeat polymorphisms in the TERT gene in lung cancer. Cancer Sci 2011;102:144-149.
45 Hsu CP, Lee LW, Shai SE, Chen CY: Clinical significance of telomerase and its associate genes expression in the maintenance of telomere length in squamous cell carcinoma of the esophagus. World J Gastroenterol 2005; 11:6941-6947.

46 Safont MJ, Gil M, Sirera R, Jantus-Lewintre E, Sanmartín E: The prognostic value of hTERT expression levels in advanced-stage colorectal cancer patients: a comparison between tissue and serum expression. Clin Transl Oncol 2011;13:396-400.

47 Suso EM, Dueland S, Rasmussen AM, Vetrhus T, Aamdal S: hTERT mRNA dendritic cell vaccination: complete response in a pancreatic cancer patient associated with response against several hTERT epitopes. Cancer Immunol Immunother 2011;60:809818.
8 Zhang B, Beeghly-Fadiel A, Long J, Zheng W: Genetic variants associated with breast-cancer risk: comprehensive research synopsis, meta-analysis, and epidemiological evidence. Lancet Oncol 2011;12:477-488.

49 Andersson S, Wallin KL, Hellström AC, Morrison LE, Hjerpe A: Frequent gain of the human telomerase gene TERC at 3q26 in cervical adenocarcinomas. Br J Cancer 2006;95: 331-338.

50 Aulmann S, Schleibaum J, Penzel R, Schirmacher P, Gebauer G: Gains of chromosome region 3q26 in intraepithelial neoplasia and invasive squamous cell carcinoma of the vulva are frequent and independent of HPV status. J Clin Pathol 2008;61:1034-1037. 